\title{
A pilot study using laser-based technique for non-invasive diagnostics of hypertensive conditions in mice
}

Karina S. Litvinova, Shakil Ahmad, Keqing Wang, llya E. Rafailov, Sergei G. Sokolovski, et al.

Karina S. Litvinova, Shakil Ahmad, Keqing Wang, Ilya E. Rafailov, Sergei G. Sokolovski, Lin Zhang, Edik U. Rafailov, Asif Ahmed, "A pilot study using laser-based technique for non-invasive diagnostics of hypertensive conditions in mice," Proc. SPIE 9689, Photonic Therapeutics and Diagnostics XII, 96893H (29 February 2016); doi: 10.1117/12.2213026

SPIE. Event: SPIE BiOS, 2016, San Francisco, California, United States 


\title{
A PILOT STUDY USING LASER-BASED TECHNIQUE FOR NON-INVASIVE DIAGNOSTICS OF HYPERTENSIVE CONDITIONS IN MICE.
}

\author{
Karina S. Litvinova ${ }^{1}$, Shakil Ahmad ${ }^{2}$, Keqing Wang ${ }^{2}$, Ilya E. Rafailov ${ }^{3}$, Sergei G. Sokolovski ${ }^{1}$, \\ Lin Zhang ${ }^{3}$, Edik U. Rafailov ${ }^{1}$, Asif Ahmed ${ }^{2}$ \\ ${ }^{1}$ Optoelectronics and Biomedical Photonics Group, Aston Institute of Photonic Technologies, \\ Birmingham, UK \\ ${ }^{2}$ Laboratory of Vascular Biology and Maternal Health, Aston Medical Research Institute, \\ Aston Medical School, Birmingham, UK \\ ${ }^{3}$ School of Engineering and Applied Science, Aston Institute of Photonic Technologies, \\ Birmingham, UK
}

\begin{abstract}
Endothelial dysfunction is directly linked to preeclampsia, a maternal hypertensive condition that is life threating for both the mother and the baby. Epidemiological studies show that women with a history of pre-eclampsia have an elevated risk for cardiovascular disease. Here we report a new non-invasive diagnostic test for preeclampsia in mice that allows us to non-invasively assess the condition of the animals during the experiment and treatment in established models of preeclampsia. A laser-based multifunctional diagnostics system (LAKK-M) was chosen to carry out non-invasive analysis of multiple parameters. The device was used to simultaneously record the microcirculatory blood flow and oxygen saturation, as well as fluorescence levels of endogenous fluorophores. Preliminary experiments were conducted on adenoviral (Ad-)- mediated overexpression of sFlt-1 (Ad-sFlt-1) to mimic preeclampsialike symptoms in mice. The recorded data displayed the ability of the LAKK-M diagnostics device to detect significant differences in perfusion measurements between the control and Ad-sFlt-1 treatment. Preliminary results provide a potential avenue to employ these diagnostics technology to monitor and aid in maintaining control of live animal conditions throughout the experiment and treatment.
\end{abstract}

Keywords: non-invasive diagnostics, preeclampsia, microcirculation, perfusion, autofluorescence, sFlt-1, NADH, FAD.

*k.litvinova@aston.ac.uk; phone +44(0)1212043703.

Photonic Therapeutics and Diagnostics XII, edited by Bernard Choi, et al., Proc. of SPIE Vol. 9689, 96893H · C 2016 SPIE · CCC code: 1605-7422/16/\$18 · doi: 10.1117/12.2213026

Proc. of SPIE Vol. $968996893 \mathrm{H}-1$ 


\section{INTRODUCTION}

Biomedical photonics is a fast growing field with a very broad spectrum of technologies and techniques for noninvasive clinical monitoring, molecular diagnostics and imaging of physiological parameters in living cells, humans, and whole organisms. Optical methods for studying biological tissues have significant advantages over other methods. These methods are used for monitoring the functional cardiac activity, operation of the vascular system, determination of blood flow and lymph flow velocity, blood volume in biotissues and the degree of their oxidation ${ }^{1}$. Laser Doppler flowmetry (LDF) is the most widely used method for observing the microcirculation, to assess the level of macro- and microcirculation in the tissue. However, the LDF technique has some limitations. Tissue reflectance oximetry (TRO) is a complimentary technique that works in real time, allowing for the study of physiological rhythms, dynamic processes in microcirculatory systems and oxygen transport in tissues. Simultaneously, the unique capability of in vivo/situ fluorescence spectroscopy (FS) to register and analyse many lifelong maintained endogenously fluorescing substances in tissues and organs can be seen as a specific niche of the technique in broader medical application. These substances largely determine the metabolism and viability of all our cells, tissues, organs and the whole body. The techniques employed by fluorescence spectroscopy are based on the analysis of induced fluorescence intensity in tissue when it is irradiated by low-intensity laser light of specific wavelengths ${ }^{2}$.

Despite the plethora of diagnostics devices available commercially (for example the LAKK-M - a multifunctional laser-based non-invasive diagnostic system), developed for tissue research and diagnostics, there is no developed and standardised methodology to monitor the real time conditions of living organisms. Furthermore, ability to monitor the effects of treatment in a non-invasive manner has not presented itself as a substantially established field. Cardiovascular diseases (CVD) and subsequent complications provide an ideal model to highlight the extent of possibility for non-invasive diagnostics device application. Currently, there are no suitable and cost effective noninvasive, pre-clinical diagnostic tools for assessing microcirculation and redox status in vivo.

Oxidative stress and endothelial dysfunction accelerate the development of preeclampsia. Loss of vascular function is considered one of the first pathological symptoms in disease progression and has been established in patients with Type I or Type II diabetes, systemic sclerosis, hypertension, as well as other CDV's. Preeclampsia is a serious, multifactorial complication of pregnancy. Numerous studies attempted to identify the risk factors for preeclampsia. At the same time, it is believed that patients with these risk factors do not necessarily develop preeclampsia. Clinical manifestations of preeclampsia develops after 20 weeks of pregnancy, and are specific to women during pregnancy and childbirth. The only radical treatment method of this pregnancy complication is delivery. Nitric oxide plays an important role in the development of normal pregnancy, increasing its synthesis in various tissues promotes adequate development of pregnancy. It is known that vascular physiological adaptation including increasing blood volume, increased throughput by reducing the vascular resistance due to nitric oxide production, occurs during normal pregnancy in 
humans. Disruption of endothelial homeostasis ${ }^{3}$, oxidative stress $^{4}$ and the loss of vascular endothelial growth factor (VEGF) activity ${ }^{5,6}$ are key contributors to the preeclampsia.

Here we describe a novel optical technique for the non-invasive assessment of preeclampsia in an in vivo mouse model relevant to oxidative stress utilising the LAKK-M to carry out the non-invasive multi-parametric analysis. The system comprises of four different lasers and is equipped with an optical probe that has eight individual fibres; four fibres serve as receivers for output radiation signals and four fibres for laser radiation delivery. The system has four channels TRO, LDF, tissue oximetry and $\mathrm{FS}^{7}$. These experimental studies evaluated the tissue blood perfusion and oximetry methods, as well as the content of coenzymes NADH and FAD in order to determine the monitoring capacity of such techniques on live animals ${ }^{8}$.

\section{MATERIALS AND METHODS}

All animal experiments were carried out using procedures approved by the Aston University Ethical Review Committee, and in accordance with the United Kingdom Animals (Scientific Procedures) Act 1986.

Female wild-type (WT) C57/black 12 mice were used in this study. Mice were housed in cages, supplied with food and water without restriction, and maintained in a 12 -h light/dark air-conditioned $\left(20-25^{\circ} \mathrm{C}\right)$ environment.

\subsection{Experimental Design}

The mice ( $\mathrm{n}=12$ ) were divided into two major groups based on the conditions of the experiment. Six animals were studied under isoflurane gas anaesthesia (now referred to as the Gas group), while the other six mice were studied under conditions without anaesthesia (now referred to as the No-Gas group). Both groups were further divided into a control group injected with empty adenovector containing the CMV promoter (Ad-CMV) and an s-Flt-1 group injected with adenoviral sFlt-1 (Ad-sFlt-1).

Measurements on the Gas group were done with the mice lying on a non-heated pad. Data recordings were taken from the ear of the anaesthetised animals. The mice from the No-Gas group had to be contained in a mouse restraint tube to reduce the effects of their movement on the recordings. Due to the lack of anaesthesia, data recordings were taken from the tails of these animals. The tails of these mice were held down to keep them extended for the duration of the measurement. 


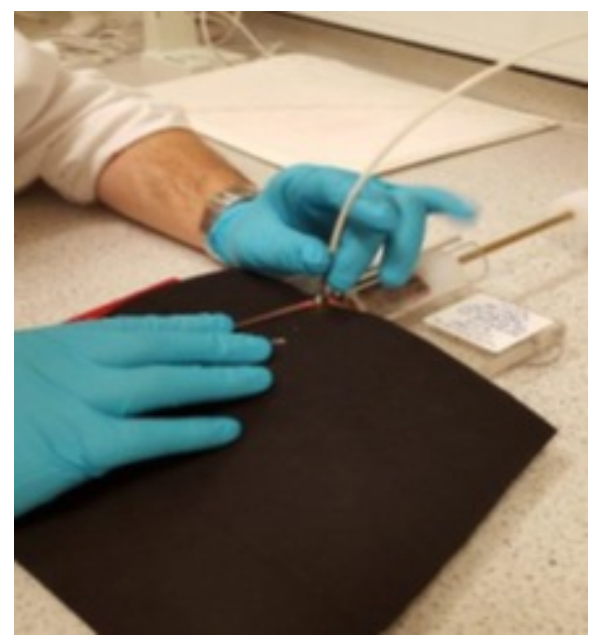

Figure 1. Study design

Figure 1 shows the study design. The LAKK-M employed an LDF channel using a $1064 \mathrm{~nm}$ wavelength Doppler laser module, a TRO module using an additional $532 \mathrm{~nm}$ and $635 \mathrm{~nm}$ wavelengths and a separate FS module with $365 \mathrm{~nm}$ and $450 \mathrm{~nm}$ wavelength sources of radiation to excite the fluorescence of NADH and FAD, respectively. An optical fibre with a $3 \mathrm{~mm}$ diameter probe was used to detect the resultant signal.

The first measurements were taken prior to the injection of Ad-CMV and Ad-sFlt-1 viruses for all mice. The next measurement was done 5 days after the viral injections. The optical probe of the LAKK-M was held in place on the ear for Gas group and the tail of the No-Gas mice (Fig 1). The perfusion, saturation and fluorescence of NADH and FAD were recorded. All LDF measurements were 20-30 seconds long, while all fluorescence measurements were around 5-10 seconds long.

\subsection{Statistics}

Data presented in the text are means \pm SD. Statistical analysis was performed using Origin 9.0. For normally distributed data, group comparisons were made by carrying out a parametric unpaired t-test. 


\section{RESULTS AND DISCUSSION}

The recorded results are displayed in Table 1 below.

Table 1

\begin{tabular}{|c|c|c|c|c|c|c|c|c|c|}
\hline \multirow{2}{*}{\multicolumn{2}{|c|}{$\begin{array}{c}\text { Mice } \\
\text { Group n }=\mathbf{3}\end{array}$}} & \multicolumn{2}{|c|}{$\mathbf{I}_{\mathrm{m}} \mathrm{Av} \pm \mathrm{SEM}$} & \multicolumn{2}{|c|}{$\mathrm{SO}_{2} \pm \mathrm{SEM}$} & \multicolumn{2}{|c|}{$\mathbf{k}_{\mathrm{NADH}} \pm \mathrm{SEM}$} & \multicolumn{2}{|c|}{$\mathrm{k}_{\mathrm{FAD}} \pm \mathrm{SEM}$} \\
\hline & & Pre & Post & Pre & Post & Pre & Post & Pre & Post \\
\hline \multirow[t]{2}{*}{ Gas } & Ad-CMV & $18.09 \pm 1.55$ & $18.02 \pm 2.86$ & $58.32 \pm 5.44$ & $64.52 \pm 12.33$ & $1.49 \pm 0.25$ & $0.89 \pm 0.11$ & $0.53 \pm 0.06$ & $0.63 \pm 0.26$ \\
\hline & Ad-sFlt-1 & $18.06 \pm 0.89$ & $13.67 \pm 0.15^{* \$}$ & $63.42 \pm 4.43$ & $58.80 \pm 13.98^{*}$ & $1.06 \pm 0.14$ & $0.43 \pm 0.05$ & $0.29 \pm 0.04$ & $0.69 \pm 0.06$ \\
\hline \multirow{2}{*}{$\begin{array}{l}\text { No- } \\
\text { Gas }\end{array}$} & Ad-CMV & $12.39 \pm 2.91$ & $14.75 \pm 1.10$ & $67.76 \pm 5.28$ & $57.64 \pm 7.18$ & $0.97 \pm 0.06$ & $0.91 \pm 0.04$ & $0.61 \pm 0.10$ & $0.49 \pm 0.09$ \\
\hline & Ad-sFlt-1 & $12.02 \pm 2.44$ & $16.66 \pm 1.92$ & $66.68 \pm 2.16$ & $57.28 \pm 4.51$ & $0.93 \pm 0.08$ & $0.92 \pm 0.01$ & $0.43 \pm 0.02$ & $0.49 \pm 0.03$ \\
\hline
\end{tabular}

As the table above shows, significant difference in blood perfusion of the Gas group of mice was found in the Ad-sFlt-1 animals after 5 days of injection, as well as in comparison to the Post- Ad-CMV animals. No significance was observed in the parameters of the No-Gas group. It is important to note that the No-Gas group displayed a significant $\mathrm{SO}_{2}$ difference in the Ad-sFlt-1 mice five days post injection. It is possible that the lack of such significance in the Gas group is due to the use of anaesthesia, which suppresses the blood oxygen saturation signal. The introduction of AdCMV into animals of both the Gas and the No-Gas groups yielded no significant differences.

Due to the differences in the experimental approach between the Gas and No-Gas groups, normalised parameters had to be calculated to compare the data. The index of oxygen saturation to perfusion of microvascular blood flow $\left(\mathrm{S}_{\mathrm{m}}\right)$, redox ratio $(\mathrm{RR})$ and the metabolic rate $(\mathrm{MR})$ were of interest for this study. They were calculated using the following equations:

$$
S m=\frac{S O 2}{I m}
$$

Where $\mathrm{SO}_{2}$ is the measured blood saturation and $\mathrm{I}_{\mathrm{m}}$ is the measured perfusion.

$$
R R=\frac{k_{N A D H}}{k_{F A D}}
$$

Where $\mathrm{k}_{\mathrm{NADH}}$ and $\mathrm{k}_{\mathrm{FAD}}$ are the fluorescence coefficients of measured NADH and FAD respectively.

$$
M R=\frac{R R}{I m}
$$

The calculated index of oxygen saturation to perfusion in microvascular blood is displayed in figure 2. No significant differences were observed in the animals of the Gas groups. As has been mentioned before, however, the NoGas group displays a significant difference in saturation. This leads to a sufficient difference in the calculated Sm parameter for the Ad-sFlt-1 treated mice of the No-Gas group. 


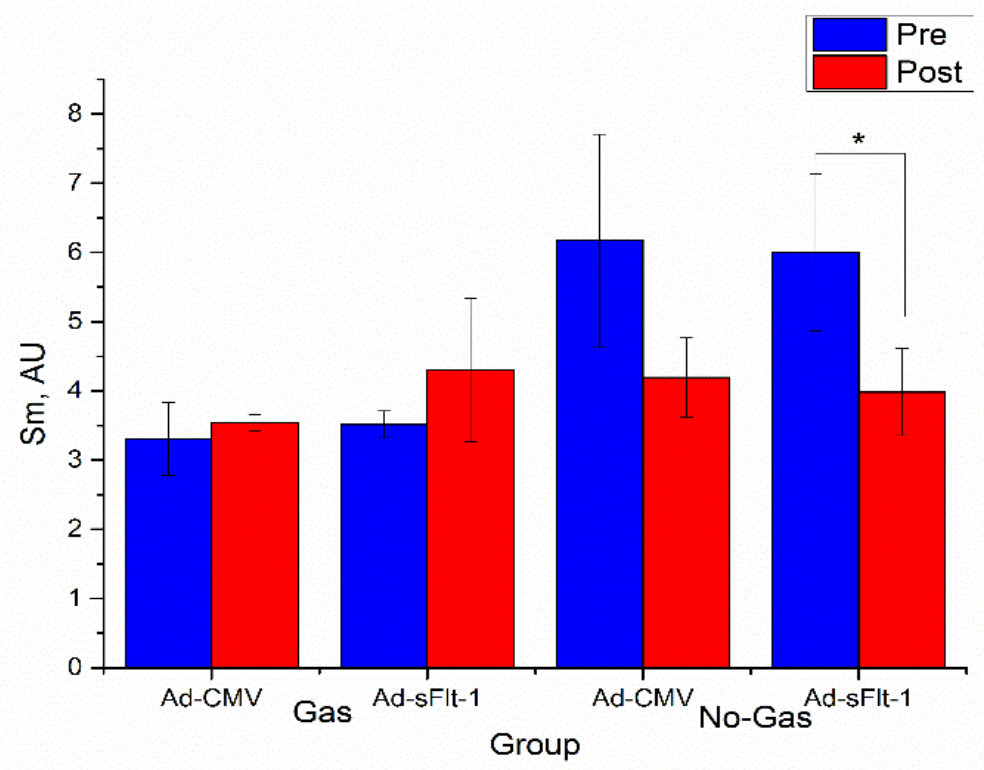

Figure 2. The comparison of the Gas and the No-Gas groups, at the start of experiment and five days after injection of CMV control and Ad-sFlt-1. Data results for calculated parameter $\mathrm{S}_{\mathrm{m}}=\mathrm{SO}_{2} / \mathrm{I}_{\mathrm{m}}$.

* Significant difference $(\mathrm{p}<0.05)$ Pre vs. Post groups in the No-Gas set of animals, calculated using a t-test.

Furthermore, the introduction of the parameter Sm allows for the comparison of data received from different areas of tissue. It normalised the oxygen saturation to the perfusion in a volume of blood measured by the probe.

Using the measured fluorescence of NADH and FAD, and the normalisation to the optical backscattering signal (k), the RR and subsequently the MR were calculated (figures 3 and 4 respectively).

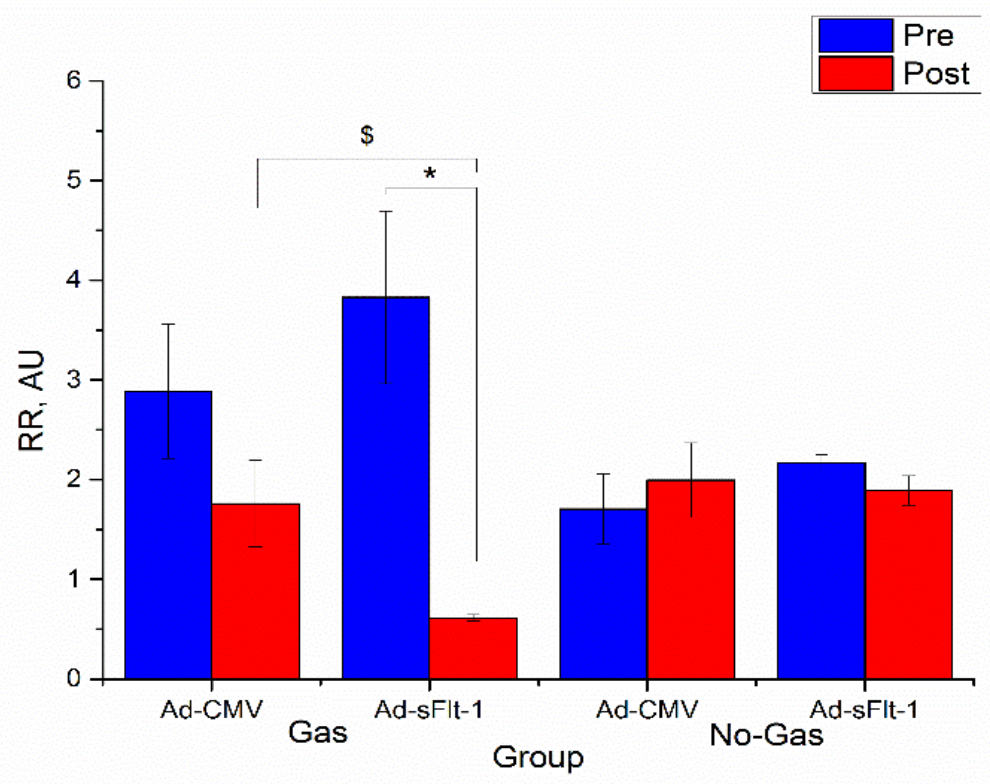

Figure 3. The comparison of the Gas and the No-Gas groups, at the start of experiment and five days after injection of CMV control and Ad-sFlt-1. Data results for calculated parameter RR.

* Significant difference $(\mathrm{p}<0.05)$ Pre vs. Post groups in the No-Gas set of animals, calculated using a t-test.

$\$$ Significant difference $(\mathrm{p}<0.05)$ Post-Control vs. Post-sFlt, calculated using a t-test. 
Interestingly, significance is pronounced in the RR parameter of the virus treated animal group. Moreover, further significant difference was noted between the five day Ad-CMV control animals and the five day Ad-sFlt-1 injected animals. This could suggest a critical disturbance in cellular metabolism (determined by the NADH/FAD ratio) due to the action of the injected Ad-sFlt-1. No significant differences were observed in the No-Gas animal group. This could potentially be explained by a variety in the measurements arising from the differing experimental approaches employed in the two groups. The measurement of ears in the Gas group allows for complete contact of the probe to the tissue due to flatness of the latter. Measurements on the tail as carried out in the No-Gas group, however, may result in incomplete contact between the probe and the round extremity. A smaller diameter of optical probe may somewhat reduce the potential variation of measuring non-flat bodies such as mouse tails.

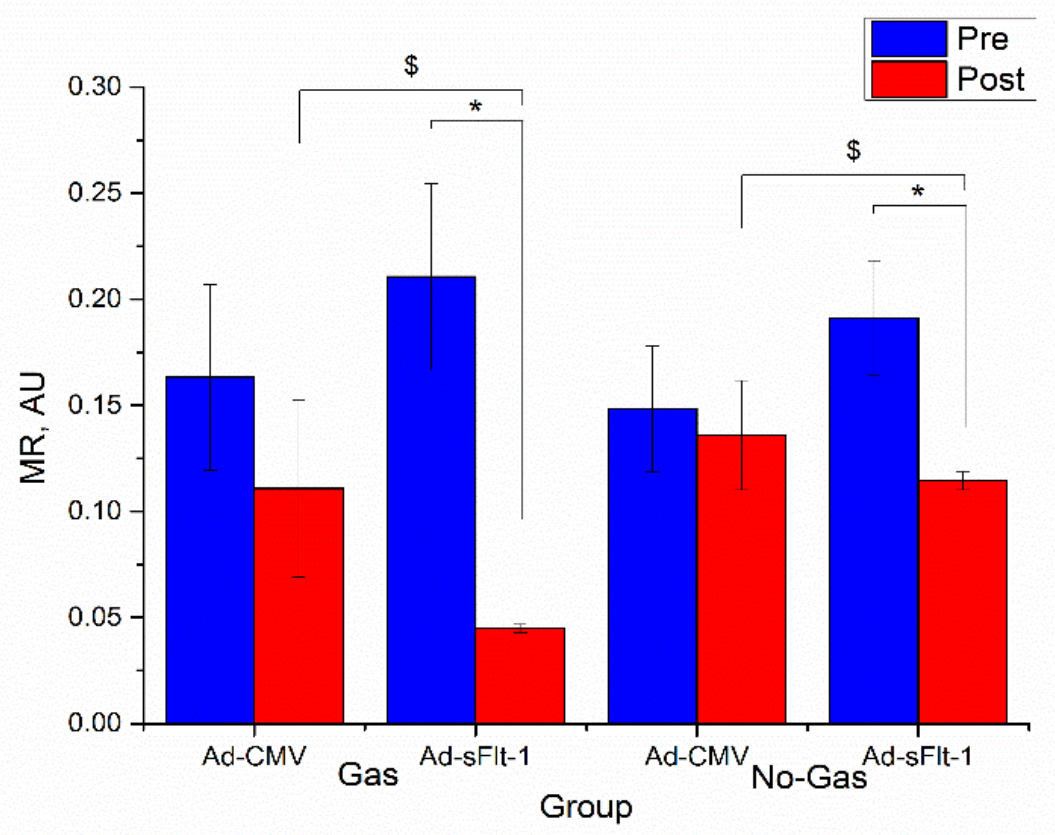

Figure 4. The comparison of the Gas and the No-Gas groups, at the start of experiment and five days after injection of CMV control and Ad-sFlt-1. Data results for calculated parameters MR.

* Significant difference $(\mathrm{p}<0.05)$ Pre vs. Post groups, calculated using a t-test.

${ }^{\$}$ Significant difference $(\mathrm{p}<0.05)$ Post-Control vs. Post-sFlt, calculated using a t-test.

Consequently, the determined tissue metabolic rate of the Gas group shows significant differences in the AdsFlt-1 treated group as well as in comparison to the Ad-CMV control group five days after treatment. It should be noted that this significance is simultaneously mirrored in the No-Gas group. Considering this, MR presents itself as an informative parameter capable of combining the blood microcirculation parameter with reduction-oxidation ratio, thus more completely representing the complexity of whole organism metabolism affected by the Ad-sFlt-1 virus.

These data further indicate the ability to monitor the condition of animals with or without anaesthetised state. As visible from all the data above, the Ad-CMV control virus did not seem to affect the status of any of the injected 
animals. As such, the monitoring is still effective, despite the difficulties arising from fluorescence measurements on the mouse tail. Based on this preliminary work, the next step is to begin experimental study of preeclampsia-like symptoms in pregnant transgenic mice.

\section{CONCLUSION}

In this paper, we demonstrated the ability of the LAKK-M to carry out real time monitoring of vital parameters of the animal under different conditions. By measuring a number of key physiological characteristics $\left(\mathrm{I}_{\mathrm{m}}, \mathrm{SO}_{2}, \mathrm{NADH}\right.$ and FAD fluorescence) and subsequently calculating further parameters ( $\mathrm{S}_{\mathrm{m}}, \mathrm{RR}$ and $\left.\mathrm{MR}\right)$, it is possible to observe the microcirculatory and metabolic changes after Ad-sFlt-1 treatment of animals as well as between virus treated and CMV control animals. Moreover, this monitoring is effective on anaesthetised and un-anaesthetised animals, providing flexibility in terms of future application.

\section{References}

[1] Tuchin, V. V., [Handbook of optical biomedical diagnostics], SPIE Press, Bellingham WA (2002).

[2] Rafailov, E.U., Litvinova, K.S., and Sokolovski, S.G., "Towards novel compact laser sources for non-invasive diagnostics and treatment," SPIE proceedings 9550, 95500G (2015).

[3] Roberts, J.M., "Endothelial dysfunction in preeclampsia.," Seminars in reproductive endocrinology 16(1), 5-15 (1998).

[4] Rodgers, G.M., Taylor, R.N., and Roberts, J.M., "Preeclampsia is associated with a serum factor cytotoxic to human endothelial cells.," American journal of obstetrics and gynecology 159(4), 908-914 (1988).

[5] Ahmed, A., and Cudmore, M.J., "Can the biology of VEGF and haem oxygenases help solve pre-eclampsia?," Biochemical Society Transactions 37(6), 1237-1242 (2009).

[6] Maynard,Karumanchi \& Ananth., "Angiogenic factors and preeclampsia.," Seminars in nephrology 31(1), 33-46 (2011).

[7] Dunaev, A. V., Sidorov, V. V., Stewart, N.A., Sokolovski, S.G., and Rafailov, E.U., "Laser reflectance oximetry and Doppler flowmetry in assessment of complex physiological parameters of cutaneous blood microcirculation," in SPIE Proc. 8572, 857205 (2013).

[8] Mayevsky, A., and Chance, B., "Oxidation-reduction states of NADH in vivo: from animals to clinical use.," Mitochondrion 7(5), 330-9 (2007). 